\title{
BMJ Open Exploring the challenges of implementing e-health: a protocol for an update of a systematic review of reviews
}

\author{
Jamie Ross, Fiona Stevenson, Rosa Lau, Elizabeth Murray
}

To cite: Ross J, Stevenson F, Lau $\mathrm{R}$, et al. Exploring the challenges of implementing e-health: a protocol for an update of a systematic review of reviews. BMJ Open 2015;5: e006773. doi:10.1136/ bmjopen-2014-006773

- Prepublication history and additional material is available. To view please visit the journal (http://dx.doi.org/ 10.1136/bmjopen-2014006773).

Received 29 September 2014 Revised 9 March 2015 Accepted 20 March 2015

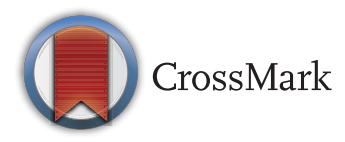

e-Health Unit, Research Department of Primary Care and Population Health, University College London, London, UK

Correspondence to Jamie Ross;

Jamie.Ross@ucl.ac.uk

\section{ABSTRACT}

Introduction: There is great potential for e-health to deliver cost-effective, quality healthcare and spending on e-health systems by governments and healthcare systems is increasing worldwide. However, the literature often describes problematic and unsuccessful attempts to implement these new technologies into routine clinical practice. To understand and address the challenges of implementing e-health, a systematic review was conducted in 2009 , which identified several conceptual barriers and facilitators to implementation. As technology is rapidly changing and new e-health solutions are constantly evolving to meet the needs of current practice, an update of this review is deemed necessary to understand current challenges to the implementation of e-health. This research aims to identify, summarise and synthesise currently available evidence, by undertaking a systematic review of reviews to explore the barriers and facilitators to implementing e-health across a range of healthcare settings.

Methods and analysis: This is a protocol for an update of a systematic review of reviews. We will search MEDLINE, EMBASE, CINAHL, PSYCINFO and The Cochrane Library for studies published between 2009 and 2014. We will check reference lists of included studies for further studies. Two authors will independently screen the titles and abstracts identified from the search; any discrepancies will be resolved by discussion and consensus. Full-text papers will be obtained and relevant reviews will be selected against inclusion criteria. Eligible reviews have to be based on the implementation of e-health technologies. Data from eligible reviews will be extracted using a data abstraction form. A thematic analysis of barriers and facilitators to e-health implementation will be conducted.

Ethics and dissemination: Ethical approval is not required. The permission of the original authors to update the review was sought and granted.

Trial registration number: PROSPERO CRD42015017661.

\section{INTRODUCTION}

The use of technology in providing and delivering healthcare is pervasive worldwide. ${ }^{12}$
Strengths and limitations of this study

- This systematic review of reviews aims to produce a comprehensive overview related to the field of implementation of e-heath; and it will not be restricted to any healthcare setting or health condition.

- Comprehensive search strategy; all citations identified from the search will be double screened.

- As in all systematic reviews, the search may not identify all relevant literature; this risk will be minimised by an inclusive search strategy and by citation-tracking all included papers for additional literature.

There are thousands of websites offering health information of varying quality used by health professionals as well as by laypersons, and online health information has become one of the most important information sources for people seeking health information in recent years. ${ }^{3}$ A survey by the Office for National Statistics reports that $43 \%$ of surveyed UK internet users have accessed health information online and this figure increases to $59 \%$ among those aged $24-35 .{ }^{4}$ In developing countries, mobile phone technologies have improved health outcomes for chronic disease conditions such as diabetes, heart disease and hypertension. ${ }^{5}$ The use of e-health, a term that describes the application of information, computer or communication technology to some aspect of health or healthcare, is viewed as integral to solving problems facing healthcare systems. ${ }^{6}$ The European Commission states that e-health will play a key role in structural reforms that are needed to ensure the sustainability of health systems while securing access to services for all citizens. ${ }^{7}$

Large-scale, national initiatives designed to coordinate e-health implementation are underway across the world and this trend is likely to increase in the future. ${ }^{8}$ Examples of e-health technologies becoming widely used 
include: management systems, such as the electronic health record (EHR), which allow the acquisition, transmission and storage of patient data; computerised decision support systems including diagnostic support, alerts and reminder systems; communication systems such as telecommunication; and information resources such as the internet. In 2002, the National Health Service (NHS) pledged £11.4 billion to reform the UK’s healthcare system, ${ }^{9}$ and this has seen the on-going introduction of many new e-health systems such as: broadband networks; systems to electronically share X-rays; the creation of an integrated EHR system; and the NHS Choices website, which provides health information to the public via the internet.

Despite the potential benefits of e-health, implementation of these systems is often reported as problematic. ${ }^{9}$ Implementation of EHR and electronic prescribing systems has lagged in most European nations as well as in the $\mathrm{USA}^{10}{ }^{10}$ costs associated with implementing e-health often spiral and time delays are reported. ${ }^{9}$ Barriers to implementation of innovations within the healthcare setting may arise at the individual, organisational and wider levels of the healthcare systems, and interact in complex and variable ways. ${ }^{11} 12$ These factors may also be innovation-specific and context-specific. Studies have described financial, legal, social and ethical barriers to implementation, arising at the organisational and individual level, including users' lack of awareness of the benefits, low e-health literacy, a shortage of evidence of cost-effectiveness and interoperability (the ability of different information technology systems and software applications to communicate, exchange data and use the information that has been exchanged) as well as security concerns. ${ }^{1}{ }^{13}$ Recognising and understanding barriers and facilitators is crucial for devising strategies and interventions to improve the widespread effective use of e-health, and addressing blockages to implementation.

A systematic review of reviews by Mair et al ${ }^{14}$ synthesised the literature on the implementation of e-health interventions in healthcare settings published up until 2009. This review found a growing emphasis on problems related to e-health systems' workability and how innovations affect organisational structures and goals. The review highlighted the need for adequate resources, particularly financial, as well as administrative support, policy support, standards and interoperability. Relatively little attention was found to be given to: e-health's effects on roles and responsibilities; risk management; ways to engage with professionals; and ensuring that the potential benefits of new technologies are made transparent through ongoing evaluation and feedback.

As the use of e-health is rapidly growing and changing, and the nature of healthcare systems are continually shifting, an update of this review was deemed timely. The Cochrane Collaboration emphasises the importance of updating systematic reviews, as evidence on a given subject is generally dynamic and continually evolving. ${ }^{15}$
This may be particularly true for the field of e-health, as the past 6 years have seen an increase in spending on information and communications technology (ICT) by healthcare systems globally and the delivery of healthcare via e-health. As well as an increase in spending, the period since the original systematic review has seen the invention and widespread adoption of technologies such as smartphones, tablets and applications, which can be used to access and deliver healthcare. The factors that promote or inhibit the implementation of e-health may also have evolved over this time, given the dynamic and expanding nature of e-health utilisation in healthcare systems, and new challenges and strategies for overcoming them may be reflected in the literature.

The aim of this review is to update a systematic review of reviews in order to summarise and synthesise published review literature on barriers and facilitators to implementing e-health in health services. The Mair et $\mathrm{al}^{14}$ systematic review was selected to update as it is the primary review in the area of e-health implementation. A review of reviews is deemed more appropriate than a further primary systematic review, as the body of literature on e-health implementation is so vast, and a secondary review of this will allow a broad overview of the literature to be synthesised, and will avoid duplicating work already undertaken by other primary reviews.

\section{METHODS AND ANALYSIS}

In updating the Mair et al ${ }^{14}$ systematic review, the methods described by those authors will be replicated, including the criteria for including and excluding studies, and the search strategy. This review will analyse data thematically.

\section{Criteria for considering studies for review}

This will be a systematic review of reviews including data from qualitative and quantitative reviews that will replicate the methodology described in the Mair et $a l^{14}$ systematic review. The eligibility criteria for study inclusion has been developed using the framework PICO, which stands for participants, interventions, comparators and outcomes (table 1).

\section{Inclusion and exclusion criteria}

Papers will be included if they meet the PICO criteria. Below is a detailed description of the inclusion and exclusion criteria for the types of studies to be considered, which is taken from the Mair $e t \mathrm{al}^{14}$ systematic review and will be used to assess the papers in this review.

Papers will be included if they are:

1. Systematic reviews: where relevant literature has been identified by means of structured search of bibliographic and other databases; where transparent methodological criteria are used to exclude papers that do not meet an explicit methodological benchmark, and which presents rigorous conclusions about outcomes. 
Table 1 PICO criteria for including studies

\begin{tabular}{ll}
\hline Population & Healthcare settings (including but not limited to: primary, intermediate, secondary, homecare) \\
& All healthcare settings will be considered \\
& Not limited by: clinical area, health concern; the type of patient who receive the e-health technology; the type of \\
health professional delivering care; or country \\
Intervention \\
Implementation of e-health \\
e-health is defined as comprising of four main domains \\
- Management systems, such as the electronic health records (EHR) that allow the acquisition, transmission \\
and storage of patient data \\
- Computerised decision support systems, including diagnostic support alerts and reminder systems \\
Communication systems, such as telecommunication, which act as an intermediary between users \\
This review is not limited to comparator studies. Where comparators are present these may comprise: standard \\
implementation processes; usual care; control; no or another implementation strategy (single or multifaceted) \\
Qualitative data on factors that inhibit or promote implementation of e-health. \\
Outcomes \\
Study type Reviews that provided descriptions of method, including systematic reviews, narrative reviews, meta-syntheses \\
or meta-ethnographies. (See inclusion and exclusion criteria for more details of these study types)
\end{tabular}

2. Narrative reviews: where relevant literature has been purposively sampled from a field of research; where theoretical or topical criteria are used to include papers on the grounds of type, relevance and perceived significance; with the aim of summarising, discussing and critiquing conclusions.

3. Qualitative metasyntheses or meta-ethnographies: where relevant literature has been identified by means of a structured search of bibliographic and other databases, where transparent methods had been used to draw together theoretical products, with the aim of elaborating and extending theory.

Papers will be excluded if they are:

1. Secondary analyses (including qualitative metasyntheses or metaethnographies) of existing data sets for the purposes of presenting cumulative outcomes from personal research programmes.

2. Secondary analyses (including qualitative metasyntheses or metaethnographies) of existing data sets for the purposes of presenting integrative outcomes from different research programmes.

3. Discussions of literature included in contributions to theory building or critique.

4. Summaries of literature for the purposes of information or commentary.

5. Editorial discussions that argue the case for a field of research or a course of action.

Where an abstract states it is a review, but there is no supporting evidence in the main paper, such as details of databases searched or criteria for selection of papers (either on methodological or theoretical grounds), the paper will be excluded.

\section{Search strategy for identification of studies}

The search strategy will be based on the following two concepts: e-health and implementation. The search strategy will include a combination of medical subject headings and free-text words. Medical subject headings referring to e-health include:

- Medical-Informatics-Applications,

- Management-Information-Systems,

- Decision-Making-Computer-Assisted,

- Diagnosis-Computer-Assisted,

- Therapy-Computer-Assisted,

- Medical-Records-Systems-Computerized,

- Medical-Order-Entry-Systems,

- Electronic-Mail,

- Videoconferencing,

- Telemedicine,

- Computer-Communication-Networks and

- Internet.

There are no thesaurus terms for implementation, so this concept will be searched for by looking for these text words in the titles, keywords or abstracts:

- Routin*,

- Normali?*,

- Integrat*,

- Facilitate*,

- Barrier*,

- Implement* and

- Adopt*.

Comprehensive electronic searches of MEDLINE, EMBASE, CINAHL, PSYCINFO and The Cochrane Library (which include Cochrane Database of Systematic reviews, Cochrane Central Register of Controlled Trials, DARE, NHSEED (NHS Economic Evaluation Database), Health Technology Assessment Database) will be conducted. The search strategy can be found in online supplementary file 1 .

The Mair et $a l^{14}$ systematic review was based on 37 reviews published between 1 January 1995 and 31 July 2009. This current review will replicate the systematic search strategy for literature published from 1 August 2009 and the 37 previously identified studies will also be included in the synthesis. 
There will be no limitation of language. Citation searches will be carried out in ISI Web of Science and reference lists of all included articles will be screened for additional literature.

\section{Selection of studies}

Titles and abstracts of all identified records will be independently double screened to ensure consistency and agreement of inclusion by JR and RL. Full text articles that are potentially eligible will be obtained, and assessed for eligibility against the prespecified inclusion and exclusion criteria. Any discrepancies between reviewers will be resolved through discussion and the involvement of a third reviewer (EM), if necessary.

\section{Data extraction and management}

A data extraction form will be developed and data will be extracted to categorise identified reviews in terms of: publication date, country of origin, aim, setting, e-health domain, databases searched, inclusion and exclusion criteria, data extraction method, quality assessment method, method of analysis/synthesis, the number of papers included in the review and factors that influence the implementation of e-health.

Each article will be carefully read by JR, and data relating to barriers and facilitators will be abstracted from the results and discussion sections of included papers. Data from the discussions will be included as they often contain interpretations from the reviewer, which may offer further insights and enhance the richness of the findings in this review of reviews. All data extraction will be checked by another reviewer (EM) who will critically examine the extraction performed by JR by checking the accuracy of data extraction, including review characteristics (eg, author, reference, aims and objectives, setting, number and type of primary studies included in the review), and the data extracted on barriers and facilitators.

\section{Data synthesis}

Once all data has been coded, it will be critically examined. If required, the coding framework will be refined and data recategorised from one theme to another, or into new themes and newly created themes will be re-configured if required. Any uncertainty about coding will be discussed between JR, FS and EM. A thematic analysis will be presented focusing on barriers and facilitators to implementation. A description will be given of any significant differences that emerge between this review and the previous systematic review. ${ }^{14}$

\section{Assessment of methodological quality}

Because we are aiming to describe and synthesise a body of qualitative literature, and not to determine an effect size, we will not conduct quality appraisal of the included studies, as it will not affect this interpretive synthesis.

\section{DISCUSSION}

This review will present up-to-date findings on factors that influence the implementation of e-health into routine practice within health settings by reviewing, summarising and synthesising the available literature. It will examine the barriers and facilitators identified from previous attempts to integrate e-health into practice and in doing so will generate possible strategies for future implementation. As an update to a previous systematic review, the findings of this review will provide an opportunity to assess whether barriers and facilitators to implementing e-health evolve or vary over time. It would seem likely, given the rapid growth and evolution of e-health technologies, that there will be differences in the factors that inhibit their implementation over time, and this will be important to recognise when devising strategies for the implementation of e-health into practice. It is also likely, however, that several of the barriers and facilitators identified in the original systematic review will remain consistent over time, which may reflect factors that are intrinsic to implementing e-health, or indeed any new innovation into healthcare settings.

A strength of this review is that it will not be limited by healthcare setting or any specific health concern and, therefore, findings will be applicable to a wide range of health services. A systematic review of reviews was selected as it allows a broad field such as this, containing many primary papers, to be synthesised. However, a potential limitation of this method is that there may be a long gap between the primary studies being conducted and the time the reviews are conducted. To mitigate the potential of missing very current insights, a scoping search of primary literature will be conducted prior to publication of this review, and if deemed appropriate, will be incorporated into the discussion of the findings.

When planning implementation, it is important to identify potential barriers and facilitators as early as possible in the process, ${ }^{16}$ to allow appropriate strategies to be formulated. In conducting this review, common challenges to e-health implementation described in the literature will be synthesised to provide learning opportunities for the implementation of e-health. Given the increased spending on ICT by healthcare systems around the world, and the often reported failures in integrating e-health systems into practice, this review will provide researchers, clinicians, commissioners and health service managers with an up-to-date understanding of the potential barriers and facilitators to successful implementation that can be applied when developing interventions and planning implementation.

\section{AMENDMENTS}

In the event of protocol amendments, the date of each amendment will be accompanied by a description of the change and the rationale.

Twitter Follow Jamie Ross at @jamieanne_dr

Acknowledgements The authors are grateful to the authors of the origina systematic review (Professor Frances Mair, Professor Carl May, Professor 
Elizabeth Murray, Dr Tracy Finch, Dr George Anderson, Professor Catherine O'Donnell, Professor Paul Wallace and Professor Frank Sullivan) for allowing us to update their work. The authors are grateful to the reviewers for their insightful comments.

Contributors JR is the guarantor. JR developed and refined the study protocol (based on the original systematic review of reviews ${ }^{14}$ ) with contributions from all co-authors (FS, EM and RL). JR prepared the manuscript. JR will undertake data collection (literature search, data extraction), analysis, interpretation and report writing, and RL and EM will act as 2nd reviewer and 3rd reviewer, respectively. FS will contribute to data interpretation and synthesis. All authors read and approved the final manuscript.

Funding The lead author (JR) is employed as part of a National Institute for Health Research (NIHR) Programme Grant for Applied Research (Grant Reference Number RP-PG-0609-10135). This literature review will be undertaken as part of the lead author's self-funded $\mathrm{PhD}$ and will contribute to the overall grant

Competing interests Professor Elizabeth Murray was a co-author on the original systematic review of reviews that will be updated. ${ }^{14}$

Provenance and peer review Not commissioned; externally peer reviewed.

Disclaimer The views expressed are those of the author(s) and not necessarily those of the NHS, the NIHR or the Department of Health.

Open Access This is an Open Access article distributed in accordance with the Creative Commons Attribution Non Commercial (CC BY-NC 4.0) license, which permits others to distribute, remix, adapt, build upon this work noncommercially, and license their derivative works on different terms, provided the original work is properly cited and the use is non-commercial. See: http:// creativecommons.org/licenses/by-nc/4.0/

\section{REFERENCES}

1. Currie WL, Seddon JJ. A cross-national analysis of eHealth in the European Union: some policy and research directions. Info Manage 2014;51:783-97.

2. Lewis T, Synowiec C, Lagomarsino G, et al. E-health in low- and middle-income countries: findings from the Center for Health Market Innovations. Bull World Health Organ 2012;90:332-40.
3. Xiao N, Sharman R, Rao HR, et al. Factors influencing online health information search: an empirical analysis of a national cancer-related survey. Decis Support Syst 2014;57:417-27.

4. Office for National Statistics. Internet Access-Households and Individuals, 2013. 2013

5. Sahu M, Grover A, Joshi A. Role of mobile phone technology in health education in Asian and African countries: a systematic review. Int J Electron Healthc 2014;7:269-86.

6. van Gemert-Pijnen J, Wynchank S, Covvey $\mathrm{H}$, et al. Improving the credibility of electronic health technologies. Bull World Health Organ 2012;90:323-323A.

7. European Commission. eHealth Action Plan 2012-2020. 2012. http://ec.europa.eu/health/ehealth/key documents/index en.htm.

8. Waterson P. Health information technology and sociotechnical systems: a progress report on recent developments within the UK National Health Service (NHS). Appl Ergon 2014;45: 150-61.

9. Public Accounts Committee. The national programme for IT in the NHS: an update on the delivery of detailed care records systems. UK: 2011 Forty-fifth Report of Session 2010-12.

10. Ben-Assuli O. Electronic health records, adoption, quality of care, legal and privacy issues and their implementation in emergency departments. Health Policy 2015;119:287-97.

11. Lau R, Stevenson F, Ong BN, et al. Addressing the evidence to practice gap for complex interventions in primary care: a systematic review of reviews protocol. BMJ Open 2014;4:e005548.

12. Sugarhood P, Wherton J, Procter R, et al. Technology as system innovation: a key informant interview study of the application of the diffusion of innovation model to telecare. Disabil Rehabil Assist Technol 2013;9:79-87.

13. Stroetmann KA, Artmann J, Dumortier J, et al. United in Diversity: legal challenges on the road towards interoperable eHealth solutions in Europe. EJBI 2012;8:3-10.

14. Mair FS, May C, O'Donnell C, et al. Factors that promote or inhibit the implementation of e-health systems: an explanatory systematic review. Bull World Health Organ 2012;90:357-64.

15. Higgins J, Green S, Scholten R. Chapter 3: Maintaining reviews: updates, amendments and feedback. Higgins JPT, Green S, eds. Cochrane Handbook for Systematic Reviews of Interventions Version 5.1.0 (updated March 2011). The Cochrane Collaboration, 2011. http://www.cochrane-handbook.org

16. Grol R, Wensing M, Eccles M. Improving patient care: the implementation of change in clinical practice. Edinburgh: Elsevier Butterworth Heinemann, 2005. 